\title{
Adições à brioflora do estado do Ceará, Brasil
}

\author{
Hermeson Cassiano de Oliveira ${ }^{1}$ \& Maria Helena Alves ${ }^{2}$
}

\section{Resumo}

(Adições à brioflora do estado do Ceará, Brasil) Foram coletadas e catalogadas 81 espécies de briófitas provenientes do município de Ubajara, localizado no Planalto da Ibiapaba, zona norte do estado do Ceará. Destas, 35 são novos registros para o estado, sendo 18 musgos distribuídas em dez famílias e 11 gêneros, 16 hepáticas em sete famílias e 14 gêneros e um antócero em uma família e um gênero. Drepanolejeunea anoplantha (Spruce) Steph., Lejeunea capensis Gottsche, Pallavicinia lyellii (Hook.) S.F. Gray, Philonotis cernua (Wilson) D.G. Griffin \& W.R. Buck, P. elongata (Dism.) H.A. Crum \& Steere, Brachymenium radiculosum (Schwägr.) Hampe, Bryum cellulare Hook., B. leptocladon Sull., B. limbatum Müll. Hal., Fissidens minutus Thwaites \& Mitt., F. ramicola Broth. e Ptychomitrium vaginatum Besch. são novas ocorrências para a Região Nordeste e Fissidens obscurocostatus Pursell é citada pela primeira vez para o Brasil. Os resultados estão organizados na forma de tabela, com informações específicas para cada espécie.

Palavras-chave: Bryophyta, Marchantiophyta, Anthocerotophyta, Ubajara, Ceará.

\section{Abstract}

(Additions to Bryoflora of the State of Ceara, Brazil) Were collected and catalogued 81 species of bryophytes proceeding from Municipal district of Ubajara, located on plateau of Ibiapaba, north zone of the state of Ceara. Of these, 35 are new records for the State, being 18 mosses distributed in ten families and 11 genera, 16 liverworts in seven families and 14 genera and one Hornwort in one family and one genus. Drepanolejeunea anoplantha (Spruce) Steph., Lejeunea capensis Gottsche, Pallavicinia lyellii (Hook.) S.F. Gray, Philonotis cernua (Wilson) D.G. Griffin \& W.R. Buck, P. elongata (Dism.) H.A. Crum \& Steere, Brachymenium radiculosum (Schwägr.) Hampe, Bryum cellulare Hook., B. leptocladon Sull., B. limbatum Müll. Hal., Fissidens minutus Thwaites \& Mitt., F. ramicola Broth. and Ptychomitrium vaginatum Besch. are news for the northeastern region and Fissidens obscurocostatus Pursell is mentioned for the first time to Brazil. The results are organized in a table with specific information for each species.

Key words: Bryophyta, Marchantiophyta, Anthocerotophyta, Ubajara, Ceará.

\section{INTRODUÇÃO}

A flora briofítica é abundante no mundo inteiro, reunindo cerca de 18.000 espécies (Shaw \& Goffinet 2000), tendo o Brasil 18\% deste número (Yano 1996). Para o Nordeste brasileiro, Pôrto (1996) listou 250 espécies de briófitas, enfatizando que os estados com maior representatividade são Bahia e Pernambuco.

Com relação ao estado do Ceará, a flora briofítica é praticamente desconhecida, sendo poucos os estudos realizados e, dentre eles, podem ser citados o de Brito \& Pôrto (2000), no qual as autoras apresentaram um catálogo com 47 espécies, contendo chaves e ilustrações para a identificação ao nível de família e o trabalho de Yano \& Pôrto (2006) onde são referidas 130 espécies como novas para o estado.
Os estudos sobre a flora cearense concernem principalmente às angiospermas, grupo de plantas predominante nos tipos vegetacionais presentes no estado. São raras as referências sobre as briófitas do Ceará, sobretudo devido à carência de coletas nas áreas de vegetação nativa. As áreas melhor exploradas, até o momento, são reduzidas considerando-se a extensão do estado, podendo-se afirmar que ainda não representam a real brioflora cearense. Os principais municípios coletados constituem encraves úmidos e subúmidos, remanescentes da mata atlântica do estado (Brito \& Pôrto 2000).

Este trabalho objetivou realizar um levantamento sobre a diversidade briofítica no município de Ubajara, contribuindo para um melhor conhecimento da brioflora do estado do Ceará.

Artigo recebido em 08/2005. Aceito para publicação em 03/2006.

${ }^{1}$ Universidade Estadual de Feira de Santana. Programa de Pós-Graduação em Botânica. Laboratório de Micologia. Av. Universitária, s/n, km 03 da BR 116, 44031-460, Feira de Santana, BA, Brasil. hermeson4@ hotmail.com

${ }^{2}$ Universidade Federal do Piauí. Curso de Licenciatura em Ciências Biológicas. Av. São Sebastião, 2819, Reis Velloso, Parnaíba, PI, Brasil. helenalves@uvanet.br 


\section{Material e Métodos}

Área de coleta - O município de Ubajara situa-se ao norte do estado do Ceará (0351'16" S, 4055'16" O), possuindo uma área de $385 \mathrm{~km}^{2}$ (Brasil 2000). Para Figueiredo (1997) a vegetação predominante na região é a caatinga, mas outros três tipos vegetacionais são encontrados na região: a floresta subperenifólia tropical plúvio-nebular (mata úmida, serrana), a floresta subcaducifólia tropical pluvial (mata seca) e o carrasco. Segundo Souza (1989), o Planalto da Ibiapaba, onde está inserido o município de Ubajara, é um dos mais importantes compartimentos geomorfológicos do território cearense. De acordo com Fernandes (1990), sua porção norte consta de condições geoclimáticas que propiciam a formação de brejos de cimeira e de encosta, caracterizados pela mata úmida, ambiente propício para o desenvolvimento de briófitas.

Material botânico - As coletas do material botânico foram efetuadas nos períodos chuvoso e seco, nas localidades do Sítio São Luís e Cachoeira do Poção, totalizando dez excursões realizadas entre os anos de 2003 e 2004. A metodologia de coleta e herborização do material briofítico seguiu Yano (1984a).

Para a identificação das amostras foram utilizados os trabalhos de Hässel de Menéndez (1962), Florschütz (1964), Fulford (1968), Hell (1969), Sehnem (1969), Schuster (1980), Ireland \& Buck (1994), Sharp et al. (1994), Ochi (1980), Oliveira-e-Silva \& Yano (2000), Lemos-Michel (2001) e Gradstein \& Costa (2003), enquanto que a distribuição geográfica brasileira das espécies foi baseada nos trabalhos de Yano (1981, 1984b, 1989, 1995), Bastos (2004), Peralta (2005) e Yano \& Pôrto (2006).
O sistema de classificação adotado foi o de Crandall-Stotler \& Stotler (2000) para hepáticas, Renzaglia \& Vaughn (2000) para antóceros e Buck \& Goffinet (2000) para os musgos. A caracterização dos tipos de substratos foi baseada em Molinaro \& Costa (2001). Os estados brasileiros estão abreviados de acordo com as normas do IBGE, na distribuição geográfica das espécies. $\mathrm{O}$ material coletado encontra-se depositado no Herbário Prof. Francisco José de Abreu Matos/ HUVA, da Universidade Estadual Vale do Acaraú /UVA, Sobral, Ceará.

\section{Resultados e Discussão}

Foram coletadas 332 amostras de briófitas, sendo 173 de hepáticas (Marchantiophyta), 154 de musgos (Bryophyta) e cinco de antóceros (Anthocerotophyta), totalizando 81 táxons. Destes, 35 são novas ocorrências para o estado. Dentre os novos registros, Drepanolejeunea anoplantha, Lejeunea capensis, Pallavicinia lyellii, Philonotis cernua, P. elongata, Brachymenium radiculosum, Bryum cellulare, B. leptocladon, B. limbatum, Fissidens minutus, $F$. ramicola e Ptychomitrium vaginatum são novas ocorrências para a Região Nordeste e Fissidens obscurocostatus é citada pela primeira vez para o Brasil. Destacam-se ainda Bryum cellulare, B. leptocladon, Fissidens minutus e Lepidopilum cubense as quais são citadas pela segunda vez para o país.

No total, foram encontradas 43 espécies de musgos distribuídas em 18 famílias e 27 gêneros, 36 espécies de hepáticas pertencentes a 10 famílias e 23 gêneros e duas espécies de antóceros em duas famílias e dois gêneros (Tab. 1). 
Tabela 1 - Brioflora encontrada no município de Ubajara, Ceará. Tipos de substrato $(C=$ corticícola, $\mathrm{EX}=$ epíxila, $\mathrm{EF}=$ epífila, $\mathrm{R}=$ rupícola, $\mathrm{T}$ = terrícola).

\begin{tabular}{|c|c|c|c|c|}
\hline Filo/Família/Espécie & Substrato & $\begin{array}{l}\text { Variação } \\
\text { altitudinal } \\
\text { no Brasil }\end{array}$ & Voucher & $\begin{array}{l}\text { Distribuição } \\
\text { geográfica }\end{array}$ \\
\hline \multicolumn{5}{|l|}{ ANTHOCEROTOPHYTA(2) } \\
\hline $\begin{array}{l}\text { ANTHOCEROTACEAE } \\
\text { Anthocerus punctatus L. }\end{array}$ & $\mathrm{T}, \mathrm{R}$ & $0-900 \mathrm{~m}$ & $\begin{array}{l}\text { Oliveira } 92 \\
148 \text { e } 150\end{array}$ & $\begin{array}{l}\text { AM, BA, CE, RJ, } \\
\text { RS e SP }\end{array}$ \\
\hline $\begin{array}{l}\text { NOTOTHYLADACEAE } \\
\text { Notothylas orbicularis (Schwein.) Sull.* }\end{array}$ & $\mathrm{T}$ & 0-950m & $\begin{array}{l}\text { Oliveira } 8 \\
146 \text { e } 159\end{array}$ & CE, FN e PE \\
\hline \multicolumn{5}{|l|}{ MARCHANTIOPHYTA(36) } \\
\hline $\begin{array}{l}\text { CALYPOGEIACEAE } \\
\text { Calypogeia laxa Gottsche \& Lindenb.* }\end{array}$ & $\mathrm{T}$ & $0-1300 \mathrm{~m}$ & $\begin{array}{l}\text { Oliveira 109, } \\
118,131,190 \\
238,239,264 \\
293 \text { e } 333\end{array}$ & $\begin{array}{l}\text { CE, ES, MG, PE, } \\
\text { RJ e SP }\end{array}$ \\
\hline C. miquelii Mont. & $\mathrm{T}$ & $0-1000 \mathrm{~m}$ & Oliveira 338 & $\begin{array}{l}\text { AM, BA, CE, MG, } \\
\text { PA, PE e RJ }\end{array}$ \\
\hline $\begin{array}{l}\text { CEPHALOZIELLACEAE } \\
\text { Cylindrocolea planifolia (Steph.) } \\
\text { R.M. Schust.* }\end{array}$ & $\mathrm{EF}$ & $0-900 \mathrm{~m}$ & Oliveira 109 & $\begin{array}{l}\text { BA, CE, MG, RJ, } \\
\text { RO, SC e SP }\end{array}$ \\
\hline $\begin{array}{l}\text { FOSSOMBRONIACEAE } \\
\text { Fossombronia porphyrorhiza (Nees) } \\
\text { Prosk.* }\end{array}$ & $\mathrm{R}$ & $0-1100 \mathrm{~m}$ & $\begin{array}{l}\text { Oliveira } 54, \\
81,86,145 \\
283,290 \text { e } 291\end{array}$ & $\begin{array}{l}\text { CE, ES, MG, MS, } \\
\text { MT, PE e RJ }\end{array}$ \\
\hline $\begin{array}{l}\text { GEOCALYCACEAE } \\
\text { Lophocolea bidentata (L.) Dumort. }\end{array}$ & $\mathrm{R}$ & $0-1500 \mathrm{~m}$ & Oliveira 123 & $\begin{array}{l}\text { AC, AM, CE, ES, } \\
\text { GO, MG, MS, PE, } \\
\text { PR, RJ, RR, RS, e SP }\end{array}$ \\
\hline $\begin{array}{l}\text { JUBULACEAE } \\
\text { Frullania riojaneirensis (Raddi) Spruce }\end{array}$ & EX,C & $0-1100 \mathrm{~m}$ & $\begin{array}{l}\text { Oliveira 132, } \\
195,245 \text { e } 262\end{array}$ & $\begin{array}{l}\text { BA, CE, DF, ES, GO, } \\
\text { MG, MT, PB, PE, } \\
\text { PR, RJ, RS, SE e SP }\end{array}$ \\
\hline $\begin{array}{l}\text { LEJEUNEACEAE } \\
\text { Acrolejeunea torulosa (Lehm. \& Lindenb.) } \\
\text { Schiffn.* }\end{array}$ & $\mathrm{C}$ & $0-800 \mathrm{~m}$ & Oliveira 149 & $\begin{array}{l}\text { AC, AL, AM, BA, } \\
\text { CE, ES, MA, MG, } \\
\text { MS, MT, PA, PR, } \\
\text { RJ, RO, RR e SP }\end{array}$ \\
\hline Bryopteris diffusa (Sw.) Nees & $\mathrm{C}$ & $0-1500 m$ & Oliveira 67 & $\begin{array}{l}\text { AL, AM, BA, CE, ES, } \\
\text { MG, MT, PA, PE, } \\
\text { PR, RJ, RS, SC e SE }\end{array}$ \\
\hline
\end{tabular}




\begin{tabular}{|c|c|c|c|c|}
\hline Filo/Família/Espécie & Substrato & $\begin{array}{l}\text { Variação } \\
\text { altitudinal } \\
\text { no Brasil }\end{array}$ & Voucher & $\begin{array}{l}\text { Distribuição } \\
\text { geográfica }\end{array}$ \\
\hline $\begin{array}{l}\text { Cheilolejeunea clausa (Nees \& Mont.) } \\
\text { R.M. Schust. }\end{array}$ & $\mathrm{R}$ & $0-900 \mathrm{~m}$ & $\begin{array}{l}\text { Oliveira } 80 \\
\text { e } 298\end{array}$ & $\begin{array}{l}\text { BA, CE, GO, MG, } \\
\text { MS, MT, PE, PR, RJ } \\
\text { e SP }\end{array}$ \\
\hline $\begin{array}{l}\text { Cheilolejeunea rigidula (Mont.) } \\
\text { R.M. Schust. }\end{array}$ & $\mathrm{C}, \mathrm{EX}$ & $0-1000 \mathrm{~m}$ & $\begin{array}{l}\text { Oliveira 09, } \\
117,217,218, \\
219,246,259, \\
301,312 \text { e } 337\end{array}$ & $\begin{array}{l}\text { AC, AM, BA, CE, } \\
\text { ES, MS, MT, PA, } \\
\text { PB, PE, PR, RJ, RR } \\
\text { e SP }\end{array}$ \\
\hline Cololejeunea subcardiocarpa Tixier* & $\mathrm{EF}$ & $0-2000 \mathrm{~m}$ & $\begin{array}{l}\text { Oliveira } 134 \\
\text { e } 251\end{array}$ & $\begin{array}{l}\text { AC, AM, BA, CE, } \\
\text { ES, MG, PE, PR, } \\
\text { RJ, SC e SP }\end{array}$ \\
\hline Diplasiolejeunea cavifolia Steph. & $\mathrm{EF}$ & $0-800 \mathrm{~m}$ & $\begin{array}{l}\text { Oliveira } 134 \\
\text { e } 251\end{array}$ & CE, PE, PR, SC e SP \\
\hline D. rudolphiana Steph.* & $\mathrm{EF}, \mathrm{C}$ & $0-800 \mathrm{~m}$ & $\begin{array}{l}\text { Oliveira } 134 \\
\text { e } 248\end{array}$ & $\begin{array}{l}\text { AC, AM, BA, CE, } \\
\text { ES, PB, PE, RJ, SC, } \\
\text { SE e SP }\end{array}$ \\
\hline $\begin{array}{l}\text { Drepanolejeunea anoplantha (Spruce) } \\
\quad \text { Steph.** }\end{array}$ & $\mathrm{EF}$ & $0-2000 \mathrm{~m}$ & Oliveira 20 & AM, CE, ES e SP \\
\hline Lejeunea capensis Gottsche** & $\mathrm{C}$ & $900-1900 \mathrm{~m}$ & Oliveira 132 & CE, MG, RJ, RS e SP \\
\hline L. flava (Sw.) Nees & $\mathrm{C}, \mathrm{EF}, \mathrm{EX}$ & $0-2400 \mathrm{~m}$ & $\begin{array}{l}\text { Oliveira 20, 38, } \\
134,151,170, \\
175,251,269 \\
320,326,328 \\
\text { e } 329\end{array}$ & $\begin{array}{l}\text { AC, AM, BA, CE, } \\
\text { DF, ES, GO, MG, } \\
\text { MS, MT, PA, PB, } \\
\text { PE, PR, RJ, RR, RS } \\
\text { e SP }\end{array}$ \\
\hline L. glaucescens Gottsche & $\mathrm{C}$ & $0-1100 \mathrm{~m}$ & $\begin{array}{l}\text { Oliveira } 17,21 \\
63 \text { e } 257\end{array}$ & $\begin{array}{l}\text { AC, BA, CE, ES, } \\
\text { MS, MT, PE, PR, } \\
\text { RJ, RR e SP }\end{array}$ \\
\hline L. grossitexta (Steph.) E. Reiner \& Goda & $\mathrm{C}$ & $0-1500 \mathrm{~m}$ & $\begin{array}{l}\text { Oliveira } 273 \\
\text { e } 294\end{array}$ & $\begin{array}{l}\text { CE, ES, MG, PR, } \\
\text { RJ, SC e SP }\end{array}$ \\
\hline L. laetevirens Nees \& Mont. & $\mathrm{C}$ & $0-1500 \mathrm{~m}$ & $\begin{array}{l}\text { Oliveira } 12,14, \\
66,94,114,138, \\
158,220,236, \\
240,253 \text { e } 322\end{array}$ & $\begin{array}{l}\text { AL, AM, BA, CE, } \\
\text { ES, FN, MG, MT, } \\
\text { PA, PB, PE, RJ, RR } \\
\text { e SP }\end{array}$ \\
\hline $\begin{array}{l}\text { Leptolejeunea elliptica (Lehm. \& } \\
\text { Lindenb.) Schiffn. }\end{array}$ & $\mathrm{EF}$ & $0-1500 \mathrm{~m}$ & $\begin{array}{l}\text { Oliveira } 29, \\
136,137,139, \\
147,224,227, \\
229 \text { e } 252\end{array}$ & $\begin{array}{l}\text { AC, AM, BA, CE, } \\
\text { DF, ES, MG, MT, } \\
\text { PA, PE, PR, RJ, RR, } \\
\text { SC e SP }\end{array}$ \\
\hline $\begin{array}{l}\text { Leucolejeunea unciloba (Lindenb.) } \\
\text { A. Evans* }\end{array}$ & $\mathrm{C}$ & $0-1300 \mathrm{~m}$ & Oliveira 103 & $\begin{array}{l}\text { AC, AM, BA, CE, } \\
\text { ES, MG, PA, PE, } \\
\text { RS, RJ, SC e SP }\end{array}$ \\
\hline $\begin{array}{l}\text { L. xanthocarpa (Lehm. \& Lindenb.) } \\
\text { A. Evans }\end{array}$ & $\mathrm{C}, \mathrm{EX}$ & $0-2500 \mathrm{~m}$ & $\begin{array}{l}\text { Oliveira } 140, \\
211,235,255, \\
271 \text { e } 279\end{array}$ & $\begin{array}{l}\text { BA, CE, ES, MG, } \\
\text { PE, RJ, RS, SC e SP }\end{array}$ \\
\hline Marchesinia brachiata (Sw.) Schiffn. & $\mathrm{C}, \mathrm{EX}, \mathrm{R}$ & $0-1700 \mathrm{~m}$ & $\begin{array}{l}\text { Oliveira } 15,127, \\
133,141,142, \\
143,144,152, \\
154,155,157 \\
215,226,250 \\
270,286 \text { e } 336\end{array}$ & $\begin{array}{l}\text { BA, CE, ES, MG, } \\
\text { PE, PR, RJ, RR, SC } \\
\text { e SP }\end{array}$ \\
\hline
\end{tabular}




\begin{tabular}{|c|c|c|c|c|}
\hline Filo/Família/Espécie & Substrato & $\begin{array}{l}\text { Variação } \\
\text { altitudinal } \\
\text { no Brasil }\end{array}$ & Voucher & $\begin{array}{l}\text { Distribuição } \\
\text { geográfica }\end{array}$ \\
\hline $\begin{array}{l}\text { Mastigolejeunea auriculata (Wilson) } \\
\text { Schiffn.* }\end{array}$ & $\mathrm{EX}$ & $0-1500 \mathrm{~m}$ & Oliveira 156 & $\begin{array}{l}\text { AC, AP, BA, CE, } \\
\text { DF, ES, GO, MG, } \\
\text { MT, PA, PR, RJ, } \\
\text { RO, RS, SC e SP }\end{array}$ \\
\hline Microlejeunea epiphylla Bischl.* & $\mathrm{EF}$ & $0-2050 \mathrm{~m}$ & $\begin{array}{l}\text { Oliveira } 30,40 \\
134,235 \text { e } 279\end{array}$ & $\begin{array}{l}\text { BA, CE, ES, MG, } \\
\text { MS, PA, PB, PE e } \\
\text { SP }\end{array}$ \\
\hline Odontolejeunea lunulata (Weber) Schiffn. & $\mathrm{EF}$ & $0-1800 \mathrm{~m}$ & $\begin{array}{l}\text { Oliveira } 22,23, \\
125,223,243, \\
251,256 \text { e } 267\end{array}$ & $\begin{array}{l}\text { AC, AM, AP, CE, } \\
\text { ES, MG, MT, PA, } \\
\text { PE, PR, RJ, RR, RS, } \\
\text { e SP }\end{array}$ \\
\hline \multicolumn{5}{|l|}{ METZGERIACEAE } \\
\hline Metzgeria aurantiaca Steph.* & EX,C & $0-1600 \mathrm{~m}$ & $\begin{array}{l}\text { Oliveira } 261, \\
295,310 \text { e } 311\end{array}$ & $\begin{array}{l}\text { AM, BA, CE, ES, } \\
\text { MG, PE, PR, RJ e SP }\end{array}$ \\
\hline M. furcata (L.) Dumort.* & $\mathrm{C}$ & $0-1500 \mathrm{~m}$ & $\begin{array}{l}\text { Oliveira } 40,75, \\
106,124,125, \\
266,285 \text { e } 308\end{array}$ & $\begin{array}{l}\text { AC, BA, CE, GO, } \\
\text { PE, PR, RJ, RS e SP }\end{array}$ \\
\hline \multicolumn{5}{|l|}{ PALLAVICINIACEAE } \\
\hline Pallavicinia lyellii (Hook.) S.F. Gray** & $\mathrm{R}, \mathrm{T}$ & $100-2000 \mathrm{~m}$ & $\begin{array}{l}\text { Oliveira } 24,42, \\
43,135 \text { e } 281\end{array}$ & $\begin{array}{l}\text { AC, AM, CE, MS, } \\
\text { PA, RJ, RS, SC e SP }\end{array}$ \\
\hline Symphyogyna aspera Steph. & $\mathrm{R}$ & $0-2200 \mathrm{~m}$ & Oliveira 280 & $\begin{array}{l}\text { AM, CE, ES, MG, } \\
\text { PE, RJ, RS, SC e SP }\end{array}$ \\
\hline \multicolumn{5}{|l|}{ PLAGIOCHILACEAE } \\
\hline $\begin{array}{l}\text { Plagiochila disticha (Lehm. \& Lindenb.) } \\
\text { Lindenb. }\end{array}$ & $\mathrm{C}, \mathrm{EF}, \mathrm{EX}$ & $0-900 \mathrm{~m}$ & $\begin{array}{l}\text { Oliveira } 31,34, \\
125,130,153, \\
213,231,232, \\
258,260,265, \\
300 \text { e } 340\end{array}$ & $\begin{array}{l}\text { AC, AM, CE, ES, } \\
\text { MG, MS, MT, PA, } \\
\text { PB, PE, RJ, RS, SC } \\
\text { e SP }\end{array}$ \\
\hline P. martiana (Nees) Lindenb. & $\mathrm{C}, \mathrm{EX}$ & $0-1100 \mathrm{~m}$ & $\begin{array}{l}\text { Oliveira } 32,35, \\
42,101,129, \\
216 \text { e } 304\end{array}$ & $\begin{array}{l}\text { CE, ES, MG, MS, } \\
\text { MT, PE, PR, RJ, RS } \\
\text { e SP }\end{array}$ \\
\hline P. micropteryx Gottsche* & $\mathrm{R}$ & $0-1000 \mathrm{~m}$ & Oliveira 120 & $\begin{array}{l}\mathrm{AC}, \mathrm{BA}, \mathrm{CE}, \mathrm{MG} \\
\mathrm{PA}, \mathrm{RJ}, \mathrm{RS} \text { e SP}\end{array}$ \\
\hline P. montagnei Nees* & EX & $0-1200 \mathrm{~m}$ & Oliveira 230 & $\begin{array}{l}\mathrm{AC}, \mathrm{AM}, \mathrm{BA}, \mathrm{CE} \\
\mathrm{ES}, \mathrm{PA}, \mathrm{PE}, \mathrm{RJ}, \mathrm{e} \mathrm{SP}\end{array}$ \\
\hline P. raddiana Lindenb. & $\mathrm{C}, \mathrm{R}$ & $0-1350 \mathrm{~m}$ & $\begin{array}{l}\text { Oliveira } 225 \\
263 \text { e } 343\end{array}$ & $\begin{array}{l}\text { AC, AM, CE, ES, } \\
\text { MG, PA, PE, PR, } \\
\text { RJ e SP }\end{array}$ \\
\hline \multicolumn{5}{|l|}{ RICCIACEAE } \\
\hline Riccia stenophylla Spruce & $\mathrm{T}$ & $0-1000 \mathrm{~m}$ & Oliveira 10 e 43 & $\begin{array}{l}\text { CE, GO, MG, RJ, } \\
\text { RS e SP }\end{array}$ \\
\hline \multicolumn{5}{|l|}{ BRYOPHYTA(43) } \\
\hline \multicolumn{5}{|l|}{ BARTRAMIACEAE } \\
\hline $\begin{array}{l}\text { Philonotis cernua (Wilson) D.G. Griffin } \\
\text { \& W.R. Buck** }\end{array}$ & $\mathrm{T}, \mathrm{C}, \mathrm{R}$ & $900-2500 \mathrm{~m}$ & $\begin{array}{l}\text { Oliveira } 102, \\
172,185,186 \\
228 \text { e } 281\end{array}$ & CE, GO, MT, RJ e SP \\
\hline P. elongata (Dism.) H.A. Crum \& Steere** & $\mathrm{T}, \mathrm{EX}$ & $0-900 \mathrm{~m}$ & Oliveira 206 e 272 & AM, CE e SP \\
\hline
\end{tabular}




\begin{tabular}{|c|c|c|c|c|}
\hline Filo/Família/Espécie & Substrato & $\begin{array}{l}\text { Variação } \\
\text { altitudinal } \\
\text { no Brasil }\end{array}$ & Voucher & $\begin{array}{l}\text { Distribuição } \\
\text { geográfica }\end{array}$ \\
\hline P. hastata (Duby) Wijk \& Margad. & $\mathrm{R}$ & $0-800 \mathrm{~m}$ & Oliveira 341 & CE, PA, RJ, RO e SP \\
\hline $\begin{array}{l}\text { P. uncinata var. glaucescens (Hornsch.) } \\
\text { Florsch. }\end{array}$ & $\mathrm{C}, \mathrm{R}$ & $0-1350 \mathrm{~m}$ & Oliveira 13 e 88 & $\begin{array}{l}\text { CE, DF, MG, MT, } \\
\text { PA, PR, RJ, RS e SP }\end{array}$ \\
\hline \multicolumn{5}{|l|}{ BRYACEAE } \\
\hline $\begin{array}{l}\text { Brachymenium radiculosum (Schwägr.) } \\
\text { Hampe** }\end{array}$ & $\mathrm{R}$ & $800-200 \mathrm{~m}$ & Oliveira 299 & $\begin{array}{l}\text { CE, ES, MG, PR, } \\
\text { RJ, RS, SC e SP }\end{array}$ \\
\hline Bryum cellulare Hook.** & $\mathrm{T}$ & $0-800 \mathrm{~m}$ & Oliveira 169 & CE e MG. \\
\hline B. leptocladon Sull.** & $\mathrm{T}$ & $0-800 \mathrm{~m}$ & Oliveira 164 & CE e GO \\
\hline B. limbatum Müll. Hal. ** & $\mathrm{R}$ & $0-800 \mathrm{~m}$ & Oliveira 98 & $\begin{array}{l}\text { CE, ES, MG, RJ, } \\
\text { RS, SC e SP }\end{array}$ \\
\hline \multicolumn{5}{|l|}{ CALYMPERACEAE } \\
\hline Calymperes erosum Müll. Hal. & EX & $0-800 \mathrm{~m}$ & Oliveira 303 & $\begin{array}{l}\text { AC, AM, AP, BA, } \\
\text { CE, MG, MT, PA, } \\
\text { PB, PE, RJ, RO e RR }\end{array}$ \\
\hline C. palisotii Schwägr. & $\mathrm{C}$ & $0-800 \mathrm{~m}$ & Oliveira 189 & $\begin{array}{l}\text { AL, AM, AP, BA, } \\
\text { CE, ES, FN, PA, PB, } \\
\text { PE, PI, PR, RJ, RN, } \\
\text { RO, RR e SE }\end{array}$ \\
\hline Octoblepharum albidum Hedw. & $\mathrm{R}, \mathrm{C}$ & $0-1200 \mathrm{~m}$ & $\begin{array}{l}\text { Oliveira } 18, \\
168 \text { e } 180\end{array}$ & $\begin{array}{l}\text { AC, AL, AM, AP, } \\
\text { BA, CE, DF, ES, FN, } \\
\text { GO, MA, MG, MS, } \\
\text { MT, PA, PE, PI, PR, } \\
\text { RJ, RN, RO, RR, RS, } \\
\text { SC, SE, SP e TO }\end{array}$ \\
\hline Syrrhopodon prolifer var. prolifer Schwägr. & r. $\mathrm{R}$ & $0-1500 \mathrm{~m}$ & $\begin{array}{l}\text { Oliveira } 46, \\
56,57,203, \\
282,289,314, \\
342 \text { e } 345\end{array}$ & $\begin{array}{l}\text { AL, AM, BA, CE, } \\
\text { DF, GO, MG, MT, } \\
\text { PA, PE, PR, RJ, RO, } \\
\text { RS, SC, SE e SP }\end{array}$ \\
\hline \multicolumn{5}{|l|}{ CRYPHAEACEAE } \\
\hline $\begin{array}{l}\text { Schoenobryum concavifolium (Griff.) } \\
\text { Gangulee* }\end{array}$ & $\mathrm{EX}, \mathrm{C}$ & $0-2000 \mathrm{~m}$ & $\begin{array}{l}\text { Oliveira } 175, \\
176,195,244 \\
\text { e } 330\end{array}$ & $\begin{array}{l}\text { BA, CE, ES, MG, } \\
\text { PE, PR, RO, SC e SP }\end{array}$ \\
\hline \multicolumn{5}{|l|}{ DICRANACEAE } \\
\hline $\begin{array}{l}\text { Campylopus heterostachys (Hampe) } \\
\text { A. Jaeger }\end{array}$ & $\mathrm{C}$ & $200-1500 \mathrm{~m}$ & Oliveira 316 & CE, MG, PI, RJ e SP \\
\hline \multicolumn{5}{|l|}{ FABRONIACEAE } \\
\hline Fabronia ciliaris (Brid.) Brid. & $\mathrm{C}$ & $0-900 \mathrm{~m}$ & $\begin{array}{l}\text { Oliveira } 71, \\
110,162,166 \\
\text { e } 167\end{array}$ & $\begin{array}{l}\text { BA, CE, ES, MG, } \\
\text { PE, PR, RJ, RS, SC } \\
\text { e SP }\end{array}$ \\
\hline \multicolumn{5}{|l|}{ FISSIDENTACEAE } \\
\hline Fissidens guianensis Mont.* & $\mathrm{R}$ & $0-900 \mathrm{~m}$ & Oliveira 122 & $\begin{array}{l}\text { AM, CE, ES, GO, } \\
\text { MS, MT, PA, PE, PI, } \\
\text { RJ, RO, SP e TO }\end{array}$ \\
\hline F. intramarginatus (Hampe) A. Jaeger & $\mathrm{C}, \mathrm{R}$ & $0-1200 \mathrm{~m}$ & $\begin{array}{l}\text { Oliveira } 01,13 \\
181 \text { e } 331\end{array}$ & $\begin{array}{l}\text { CE, ES, MG, MS, } \\
\text { MT, PR, RJ, SC e SP }\end{array}$ \\
\hline F. minutus Thwaites \& Mitt.** & $\mathrm{R}$ & $0-800 \mathrm{~m}$ & $\begin{array}{l}\text { Oliveira } 208, \\
284,332 \text { e } 334\end{array}$ & $\mathrm{CE}$ e GO \\
\hline
\end{tabular}




\begin{tabular}{|c|c|c|c|c|}
\hline Filo/Família/Espécie & Substrato & $\begin{array}{l}\text { Variação } \\
\text { altitudinal } \\
\text { no Brasil }\end{array}$ & Voucher & $\begin{array}{l}\text { Distribuição } \\
\text { geográfica }\end{array}$ \\
\hline F. obscurocostatus Pursell *** & $\mathrm{C}$ & ca. $900 \mathrm{~m}$ & Oliveira 161 & $\mathrm{CE}$ \\
\hline F. prionodes Mont. & $\mathrm{R}, \mathrm{T}$ & $0-1500 \mathrm{~m}$ & $\begin{array}{l}\text { Oliveira } 82,87 \\
111,165 \text { e } 338\end{array}$ & $\begin{array}{l}\text { AM, BA, CE, GO, } \\
\text { MG, MT, PA, PB, } \\
\text { PE, PI, PR, RJ, RO, } \\
\text { RR, RS e SP }\end{array}$ \\
\hline F. radicans Mont. & $\mathrm{C}$ & $0-800 \mathrm{~m}$ & Oliveira 242 & $\begin{array}{l}\text { BA, CE, ES, PA, } \\
\text { PB, PE, PI, PR, RJ, } \\
\text { RS e SP }\end{array}$ \\
\hline F. ramicola Broth.** & $\mathrm{R}$ & $0-800 \mathrm{~m}$ & Oliveira 287 & $\mathrm{AM}, \mathrm{CE}$ e ES \\
\hline F. serratus Müll. Hal.* & $\mathrm{T}$ & $0-900 \mathrm{~m}$ & Oliveira 163 & $\begin{array}{l}\text { CE, MG, MT, PE } \\
\text { e SP }\end{array}$ \\
\hline F. zollingeri Mont. & $\mathrm{T}$ & $0-800 \mathrm{~m}$ & Oliveira 201 & $\begin{array}{l}\text { AC, AM, BA, CE, } \\
\text { ES, FN, GO, MA, } \\
\text { MG, MS, PA, PB, } \\
\text { PE, PI, PR, RJ, RO, } \\
\text { SC, SP, e TO }\end{array}$ \\
\hline \multicolumn{5}{|l|}{ HYPNACEAE } \\
\hline Isopterygium tenerum (Sw.) Mitt. & EX & $0-1350 \mathrm{~m}$ & Oliveira 202 & $\begin{array}{l}\text { AC, AM, BA, CE, } \\
\text { ES, GO, MG, MT, } \\
\text { PA, PB, PE, PR, RJ, } \\
\text { RR, RS, SC e SP }\end{array}$ \\
\hline \multicolumn{5}{|l|}{ LEUCOBRYACEAE } \\
\hline Leucobryum crispum Müll. Hal.* & $\mathrm{T}$ & $0-1800 \mathrm{~m}$ & Oliveira 105 & $\begin{array}{l}\text { AM, BA, CE, ES, } \\
\text { GO, MG, MT, PR, } \\
\text { RJ, RR, RS, SC e SP }\end{array}$ \\
\hline Ochrobryum gardneri (Müll. Hal.) Lindb. & $\mathrm{C}$ & $0-1200 \mathrm{~m}$ & Oliveira 344 & $\begin{array}{l}\text { AM, BA, CE, GO, } \\
\text { MG, MT, PA, PE, } \\
\text { RJ, RO e SP }\end{array}$ \\
\hline \multicolumn{5}{|l|}{ ORTHOTRICHACEAE } \\
\hline $\begin{array}{l}\text { Macromitrium punctatum (Hook. \& Grev.) } \\
\text { Brid. }\end{array}$ & $\mathrm{R}$ & $0-2100 \mathrm{~m}$ & Oliveira 191 & $\begin{array}{l}\text { AM, BA, CE, ES, } \\
\text { GO e RJ }\end{array}$ \\
\hline \multicolumn{5}{|l|}{ PILOTRICHACEAE } \\
\hline Lepidopilum cubense (Sull.) Mitt.* & EX & $0-800 \mathrm{~m}$ & Oliveira 207 & $\mathrm{CE}$ e BA \\
\hline \multicolumn{5}{|l|}{ POTTIACEAE } \\
\hline Barbula agraria Hedw. & $\mathrm{R}$ & $0-800 \mathrm{~m}$ & $\begin{array}{l}\text { Oliveira } 192, \\
196 \text { e } 313\end{array}$ & $\begin{array}{l}\text { AC, AM, BA, CE, } \\
\text { FN, MG, PA, PB, } \\
\text { PE, RJ e SP }\end{array}$ \\
\hline Hyophila involuta (Hook.) A. Jaeger & $\mathrm{R}$ & $0-700 \mathrm{~m}$ & Oliveira 62 e 197 & $\begin{array}{l}\text { AM, BA, CE, DF, } \\
\text { ES, GO, MS, PA, } \\
\text { PB, PE, PI, PR, RJ, } \\
\text { RO, RS e SP }\end{array}$ \\
\hline \multicolumn{5}{|l|}{ PTEROBRYACEAE } \\
\hline $\begin{array}{l}\text { Henicodium geniculatum (Mitt.) } \\
\text { W.R. Buck* }\end{array}$ & $\mathrm{C}, \mathrm{EX}$ & $0-900 \mathrm{~m}$ & $\begin{array}{l}\text { Oliveira } 97,171, \\
183,187,193, \\
194,212,214, \\
237,247,249, \\
276,292 \text { e } 323\end{array}$ & $\begin{array}{l}\text { AC, AL, AM, AP, } \\
\text { BA, CE, DF, ES, } \\
\text { GO, MT, PA, PE, } \\
\text { RJ, RO, RS e SP }\end{array}$ \\
\hline
\end{tabular}




\begin{tabular}{|c|c|c|c|c|}
\hline Filo/Família/Espécie & Substrato & $\begin{array}{l}\text { Variação } \\
\text { altitudinal } \\
\text { no Brasil }\end{array}$ & Voucher & $\begin{array}{l}\text { Distribuição } \\
\text { geográfica }\end{array}$ \\
\hline Jaegerina scariosa (Lorentz) Arz. & EX & $0-1100 \mathrm{~m}$ & Oliveira 05 & $\begin{array}{l}\text { AL, AM, CE, DF, } \\
\text { ES, GO, MG, MS, } \\
\text { MT, PA, PE, RJ e SP }\end{array}$ \\
\hline Orthostichopsis tetragona (Hedw.) Broth. & $\mathrm{C}$ & $0-900 \mathrm{~m}$ & $\begin{array}{l}\text { Oliveira } 01,16 \\
\text { e } 278\end{array}$ & AP, CE, MG, PA e SE \\
\hline \multicolumn{5}{|l|}{ PTYCHOMITRIACEAE } \\
\hline Ptychomitrium vaginatum Besch.** & $\mathrm{R}$ & $0-1200 \mathrm{~m}$ & $\begin{array}{l}\text { Oliveira } 306, \\
319 \text { e } 335\end{array}$ & $\begin{array}{l}\text { CE, PR, RJ, RS, SC } \\
\text { e SP }\end{array}$ \\
\hline \multicolumn{5}{|l|}{ RACOPILACEAE } \\
\hline Racopilum tomentosum (Hedw.) Brid. & $\mathrm{EX}, \mathrm{C}, \mathrm{R}$ & $0-2700 \mathrm{~m}$ & $\begin{array}{l}\text { Oliveira } 02,25, \\
26,27,108, \\
184,199 \text { e } 277\end{array}$ & $\begin{array}{l}\text { AC, AM, BA, CE, } \\
\text { DF, ES, GO, MG, } \\
\text { MS, MT, PA, PE, } \\
\text { PR, RJ, RO, RS, SC } \\
\text { e SP }\end{array}$ \\
\hline \multicolumn{5}{|l|}{ SEMATOPHYLLACEAE } \\
\hline $\begin{array}{l}\text { Acroporium estrellae (Müll. Hal.) } \\
\text { W.R. Buck \& Schäf.-Verw. }\end{array}$ & EX & $0-1900 \mathrm{~m}$ & Oliveira 182 & $\begin{array}{l}\text { CE, MG, PA, RJ, } \\
\text { RS, SC e SP }\end{array}$ \\
\hline A. pungens (Hedw.) Broth. & $\mathrm{R}$ & $0-1200 \mathrm{~m}$ & $\begin{array}{l}\text { Oliveira } 204 \\
\text { e } 325\end{array}$ & $\begin{array}{l}\text { AM, AP, BA, CE, } \\
\text { ES, MG, PR, RJ, } \\
\text { RR, SC e SP }\end{array}$ \\
\hline $\begin{array}{l}\text { Pterogonidium pulchellum (Hook.) } \\
\text { Müll. Hal.* }\end{array}$ & $\mathrm{C}, \mathrm{R}, \mathrm{EX}$ & $0-900 \mathrm{~m}$ & $\begin{array}{l}\text { Oliveira } 33,78, \\
116,177,178, \\
179,198,222, \\
268,305,318 \\
\text { e } 327\end{array}$ & $\begin{array}{l}\text { AM, BA, CE, PA, } \\
\text { PE, RJ e SP }\end{array}$ \\
\hline $\begin{array}{l}\text { Sematophyllum subpinnatum (Brid.) } \\
\text { E. Britton }\end{array}$ & $\mathrm{C}, \mathrm{R}, \mathrm{EX}$ & $0-1500 \mathrm{~m}$ & $\begin{array}{l}\text { Oliveira } 39,76, \\
91,115,173, \\
174,200,210, \\
233,234,239, \\
254,275,296, \\
297,302,307, \\
309,317 \text { e } 321\end{array}$ & $\begin{array}{l}\text { AC, AM, BA, DF, } \\
\text { CE, ES, MS, PA, } \\
\text { PE, RJ e SP }\end{array}$ \\
\hline S. subsimplex (Hedw.) Mitt. & $\mathrm{C}, \mathrm{R}, \mathrm{EX}$ & $0-2000 \mathrm{~m}$ & $\begin{array}{l}\text { Oliveira } 59,90 \\
93,119,209, \\
221,241,288 \\
315, \text { e } 339\end{array}$ & $\begin{array}{l}\text { AC, AM, AP, BA, } \\
\text { CE, DF, ES, GO, MA, } \\
\text { MG, MS, MT, PA, } \\
\text { PB, PE, PI, PR, RJ, } \\
\text { RO, RR, RS, SC, SE } \\
\text { e SP }\end{array}$ \\
\hline $\begin{array}{l}\text { SPLACHNOBRYACEAE } \\
\text { Splachnobryum obtusum (Brid.) } \\
\text { Müll. Hal. }\end{array}$ & $\mathrm{T}$ & $0-900 \mathrm{~m}$ & $\begin{array}{l}\text { Oliveira } 188 \\
\text { e } 205\end{array}$ & $\begin{array}{l}\text { AC, AL, AM, CE, } \\
\text { FN, GO, MS, RS e SP }\end{array}$ \\
\hline $\begin{array}{l}\text { STEREOPHYLLACEAE } \\
\text { Eulacophyllum cultelliforme (Sull.) } \\
\text { W.R. Buck \& Ireland* }\end{array}$ & $\mathrm{R}$ & $0-900 \mathrm{~m}$ & $\begin{array}{l}\text { Oliveira } 121 \\
\text { e } 324\end{array}$ & $\begin{array}{l}\text { AM, CE, ES, MG, } \\
\text { MS, MT, PB, PE, } \\
\text { PR, RJ, SE e SP }\end{array}$ \\
\hline
\end{tabular}

* Novas ocorrências para o estado. ** Novas ocorrências para o Nordeste. *** Nova ocorrência para o Brasil. 
Dentre as hepáticas, a família mais representativa foi Lejeuneaceae com 20 espécies, seguida por Plagiochilaceae com cinco, Calypogeiaceae, Metzgeriaceae e Pallaviciniaceae com duas e Cephaloziellaceae, Fossobroniaceae, Geocalycaceae, Jubulaceae e Ricciaceae com uma espécie cada.

Com relação aos musgos, a família com o maior número de espécies foi Fissidentaceae com nove táxons, seguida por Sematophyllaceae com cinco, Bartramiaceae, Bryaceae e Calymperaceae com quatro, Pterobryaceae com três, Leucobryaceae e Pottiaceae com duas e Cryphaeaceae, Dicranaceae, Fabroniaceae, Hypnaceae, Orthotrichaceae, Pilotrichaceae, Ptycomitriaceae, Racopilaceae, Splachnobryaceae e Stereophyllaceae com uma espécie.

Para antóceros são citadas as famílias Anthocerotaceae e Notothyladaceae com uma espécie cada.

Brito \& Pôrto (2000) em levantamento feito para regiões serranas, principalmente na Serra de Maranguape, citam 47 espécies para o estado do Ceará sendo 11 hepáticas e 36 musgos distribuídos em sete e 18 famílias respectivamente. Dentre as famílias estudadas pelas autoras Calypogeiaceae, Jubulaceae, Lejeuneaceae, Ricciaceae, Fissidentaceae, Leucobryaceae, Calymperaceae, Dicranaceae, Pottiaceae, Bryaceae, Splachnobryaceae, Orthotrichaceae, Pterobryaceae, Racopilaceae, Fabroniaceae, Stereophyllaceae, Hypnaceae e Sematophyllaceae possuem representantes comuns a este trabalho, entretanto as famílias Anthocerotaceae, Notothyladaceae, Cephaloziellaceae, Fossombroniaceae,
Geocalycaceae, Metzgeriaceae, Pallaviciniaceae, Plagiochilaceae, Cryphaeaceae, Pilotrichaceae e Ptychomitriaceae não citadas pelas autoras, foram observadas nesta pesquisa.

Muitas das famílias encontradas possuem distribuição geográfica ampla, consideradas cosmopolitas (Bryaceae, Dicranaceae e Orthotrichaceae) ou apresentam distribuição concentrada nas regiões temperadas e tropicais (Calymperaceae, Metzgeriaceae, Jubulaceae e Plagiochilaceae), ou ainda, têm nítida predominância nos trópicos do Novo e do Velho Mundo (Lejeuneaceae) (Pôrto et al. 2004).

No município de Ubajara as briófitas colonizam diversos tipos de substrato: troncos e ramos vivos (corticícola), troncos em decomposição (epíxila), superfícies de rochas (rupícola), solo (terrícola) e folhas (epifila) (Tab. 2). Dentre os 81 táxons, 24 (29\%) não apresentaram preferência por substrato, crescendo sobre mais de um tipo, sendo troncos e ramos vivos os mais colonizados.

Os resultados demonstram a significativa e, ainda, pouco conhecida diversidade de briófitas existente no estado. Principalmente, no que diz respeito ao Planalto da Ibiapaba, onde poucas coletas são citadas na literatura.

Levando-se em consideração o número total de briófitas citadas para o Brasil (3240 spp) e, mais especificamente para o Nordeste (250 spp.), verifica-se a significativa relevância dos resultados alcançados, demonstrando o estado do Ceará como possuidor de $6,5 \%$ da diversidade briofítica citada a nível nacional e $85,2 \%$ no parâmetro regional.

Tabela 2 - Ocorrência dos táxons nos substratos encontrados.

\begin{tabular}{lllll}
\hline Substratos & $\mathbf{N}^{\circ}$ táxons & \% & Exclusivas & \% \\
\hline Rupícola (R) & 31 & 38 & 18 & 22 \\
Corticícola (C) & 35 & 43 & 15 & 18 \\
Epíxila (EX) & 22 & 27 & 7 & 8 \\
Terrícola (T) & 15 & 18 & 10 & 12 \\
Epifila (EF) & 10 & 12 & 7 & 8 \\
\hline Total & 113 & & 57
\end{tabular}


Unindo-se os resultados deste e de trabalhos anteriores observa-se a atualização dos dados da brioflora citada para o Ceará até o momento, contabilizando-se 213 espécies. Brito \& Pôrto (2000) contribuíram citando 47 espécies, Yano \& Pôrto (2006) acrescentaram 131 novos registros e o presente trabalho adiciona 35 novas ocorrências ao estado, contribuindo de forma efetiva para a divulgação e conhecimento da brioflora do Ceará e do Brasil.

\section{Agradecimentos}

Os autores agradecem à Fundação Cearense de Apoio ao Desenvolvimento Científico e Tecnológico/FUNCAP pela Bolsa de Iniciação Científica fornecida ao primeiro autor; à Dra. Olga Yano do Instituto de Botânica - IBt, da Secretaria do Meio Ambiente do Estado de São Paulo pela colaboração na identificação e confirmação das espécies, uso dos equipamentos do laboratório e de sua biblioteca particular e a Denilson Fernandes Peralta do IBt pelo apoio e amizade durante as identificações das amostras.

\section{REFERÊNCIAS BIBLIOGRÁFICAS}

Bastos, C. J. P. 2004. Lejeuneaceae (Marchantiophyta) no estado da Bahia, Brasil. Tese de Doutorado. Universidade de São Paulo, São Paulo.

Brasil. 2000. Ministério do Meio Ambiente/ PNMA II. Diagnóstico da Gestão Ambiental nas Unidades da Federação Ceará. Fortaleza, 113p.

Brito, A. E. R. M. \& Pôrto, K. C. 2000. Guia de estudos de briófitas: briófitas do Ceará. Edições UFC: 1-66.

Buck, W. R. \& Goffinet, B. 2000. Morphology and Classification of Mosses. In: Shaw, A. J. \& Goffinet, B. (eds.). Bryophyte Biology. University Press, Cambridge. Pp. 71-123.

Crandall-Stotler, B. \& Stotler, R. E. 2000. Morphology and classification of the Marchantiophyta. In: Shaw, A. J. \&
Goffinet, B. (eds.). Bryophyte Biology, University Press, Cambridge. Pp. 21-70.

Fernandes, A. 1990. Temas fitogeográficos. Stylos Comunicações, Fortaleza, 116p.

Figueiredo, M. A. 1997. A cobertura vegetal do Ceará (Unidades Fitoecológicas). In: Atlas do Ceará. IPLANCE, Fortaleza, 65p.

Florschütz, P. A. 1964. The mosses of Suriname. E. J. Brill, Leiden, 271p.

Fulford, M. H. 1968. Manual of the leafy Hepaticae of Latin America. Part III. Memoirs of the New York Botanical Garden 11(3): 277-392.

Gradstein, S. R. \& Costa, D. P. 2003. The Hepaticae and Anthocerotae of Brazil. Memoirs of the New York Botanical Garden 87: 1-318.

Hässel de Menéndez, G. G. 1962. Estudio de las Anthocerotales y Marchantiales de la Argentina. Opera Lilloana 7: 1-297.

Hell, K. G. 1969. Briófitas talosas dos arredores da cidade de São Paulo (Brasil). Boletim da Faculdade de Filosofia e Ciências de São Paulo, Botânica 25: 1190.

Ireland, R. R. \& Buck, W. R. 1994. Stereophyllaceae. Flora Neotropica, monograph 65: 1-49.

Lemos-Michel, E. 2001. Hepáticas epifíticas sobre o pinheiro-brasileiro no Rio Grande do Sul. Editora da Universidade/ Universidade Federal do Rio Grande do Sul, Porto Alegre, 191p.

Molinaro, L. C. \& Costa, D. P. 2001. Briófitas do arboreto do Jardim Botânico do Rio de Janeiro. Rodriguesia 52(81): 107-124.

Ochi, H. 1980. A revision of the Neotropical Bryoidae, Musci. First Part. The Journal of the Faculty of Education Tottori University. nat. sci. 29(2): 49-54.

Oliveira e Silva, M. I. M. N. \& Yano, O. 2000. Anthocerotophyta e Hepatophyta de Mangaratiba e Angra dos Reis, Rio de Janeiro, Brasil. Boletim do Instituto de Botânica 13: 1-102.

Peralta, D. F. 2005. Musgos (Bryophyta) do Parque Estadual da Ilha Anchieta (PEIA), 
São Paulo, Brasil. Dissertação de Mestrado. Instituto de Botânica, São Paulo.

Pôrto, K. C. 1996. Briófitas. In: Sampaio, E. V. S. B.; Mayo, S. J. \& Barbosa, M. R. V. (eds.). Pesquisa botânica nordestina: progresso e perspectivas. Sociedade Botânica do Brasil/Seção Regional de Pernambuco, Recife. Pp. 97-109.

; Germano, S. R. \& Borges, G. M. 2004. Avaliação dos Brejos de Altitude de Pernambuco e Paraíba, quanto à diversidade de Briófitas, para a conservação. In: Pôrto, K. C.; Cabral, J. J. P. \& Tabarelli, M. (eds.). Brejos de Altitude em Pernambuco e Paraíba. Universidade Federal de Pernambuco. 79-97.

Renzaglia, K. S. \& Vaughn, K. C. 2000. Anatomy, development and classification of hornworts. In: Shaw, J. \& Goffinet, B. (eds.). Bryophyte Biology. Cambridge University Press, Cambridge. 1-20.

Schuster, R. M. 1980. The Hepaticae and Anthocerotae of North America. vol. 4. Columbia University Press, New York.

Sehnem, S. J. A. 1969. Musgos sul-brasileiros. Pesquisas, sér. Botânica 27: 1-36.

Sharp, A. J.; Crum, H. \& Eckel, P. M. (eds). 1994. The moss flora of Mexico. Memoirs of the New York Botanical Garden 69: 11113.
Shaw, A. J. \& Goffinet, B. 2000. Bryophyte Biology. Cambridge University Press, Cambridge, 476p.

Souza, M. J. N. 1989. Geomofologia. In: Atlas do Ceará. IPLANCE, Fortaleza. Pp. 1415.

Yano, O. 1981. A checklist of Brazilian mosses. The Journal of the Hattori Botanical Laboratory 50: 279-456. 1984a. Briófitas. In: Fidalgo, O. \& Bononi, V. L. R. (coords). Técnicas de coleta, preservação e herborização de material botânico. Instituto de Botânica, São Paulo. Manual 4: 27-30.

1984b. Checklist of Brazilian liverworts and hornworts. The Journal of the Hattori Botanical Laboratory 56: 481548.

1989. An additional checklist of Brazilian bryophytes. The Journal of the Hattori Botanical Laboratory 66: 371434.

1995. A new additional annotated checklist of Brazilian bryophytes. The Journal of the Hattori Botanical Laboratory 78: 137-182.

1996. A checklist of Brazilian bryophytes. Boletim do Instituto de Botânica de São Paulo 10: 47-232.

\& Pôrto, K. C. 2006. Diversidade das briófitas das matas serranas do Ceará, Brasil. Hoehnea 33(1): 7-39. 